\title{
Spin torque and heating effects in current-induced domain wall motion probed by transmission electron microscopy
}

\author{
F. Junginger, ${ }^{\text {a) }}$ M. Kläui, ${ }^{\text {b) }}$ D. Backes,${ }^{\text {c) }}$ and U. Rüdiger \\ Fachbereich Physik, Universität Konstanz, Universitätsstraße 10, 78457 Konstanz, Germany \\ T. Kasama and R. E. Dunin-Borkowski ${ }^{\text {d) }}$ \\ Department of Materials Science and Metallurgy, University of Cambridge, Pembroke Street, \\ Cambridge CB2 3QZ, United Kingdom \\ L. J. Heyderman \\ Laboratory for Micro- and Nanotechnology, Paul Scherrer Institut, 5232 Villigen PSI, Switzerland \\ C. A. F. Vaz ${ }^{\mathrm{e})}$ and J. A. C. Bland \\ Cavendish Laboratory, University of Cambridge, Madingley Road, Cambridge CB3 OHE, United Kingdom
}

(Received 21 December 2006; accepted 24 January 2007; published online 28 March 2007)

\begin{abstract}
Observations of domain wall motion and transformations due to injected current pulses in permalloy zigzag structures using off-axis electron holography and Lorentz microscopy are reported. Heating on membranes leads to thermally activated random behavior at low current densities and by backcoating the SiN membranes with Al, heating effects are significantly reduced. A set of indicators is devised to separate unambiguously spin torque effects from heating and it is shown that by using the Al layer the structures are sufficiently cooled to exhibit current-induced domain wall motion due to spin torque. (C) 2007 American Institute of Physics. [DOI: 10.1063/1.2709989]
\end{abstract}

Domain walls and reversal by domain wall motion have recently become the focus of interest by allowing us to address fundamental physical questions, such as the geometry dependent spin structure, ${ }^{1}$ pinning of domain walls at constrictions, ${ }^{2}$ and the details of the domain wall propagation processes. ${ }^{3}$ Additionally, devices based on domain walls have been suggested for storage and logic. Recently currentinduced domain wall motion ${ }^{3-6}$ has been proposed as an alternative to the use of conventional external magnetic fields, therefore opening up a route for simple device fabrication, where no field-generating strip lines are necessary. While current-induced domain wall motion is experimentally well established, ${ }^{3,5,6}$ the underlying theory of interaction between current and magnetization is still controversial. Different approaches have been suggested in the ballistic as well as in the diffusive limit. ${ }^{7}$ The assumption that the spin of the charge carriers follows the local magnetization leads to the introduction of an adiabatic torque into the Landau-Lifshitz-Gilbert equation of magnetization dynamics. ${ }^{7}$ Motivated by large discrepancies between experiment and theory, a nonadiabatic term was introduced. ${ }^{8,9}$ While all these theories qualitatively predict domain wall motion in the electron flow direction, quantitative estimates of the velocities and, in particular, the domain wall transformations strongly depend on the formalism.

Additionally, due to the high current densities, strong heating occurs, which can be detrimental for the structures and the physical mechanisms involved. So for applications it is a key requirement to devise ways for efficient cooling,

\footnotetext{
a) Also at Department of Materials Science and Metallurgy, University of Cambridge, Pembroke Street, Cambridge CB2 3QZ, U.K.

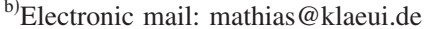

${ }^{c)}$ Also at Laboratory for Micro- and Nanotechnology, Paul Scherrer Institut, 5232 Villigen PSI, Switzerland.

d) Also at: Center for Electron Nanoscopy, DTU, DK-2800 Denmark.

${ }^{\text {e) }}$ Currently at Applied Physics, Yale University, New Haven, Connecticut 06520.
}

which means that first the heat dissipation paths need to be understood. Furthermore, domain wall transformations have been shown to originate not only from spin torque effects ${ }^{6}$ but also from thermal excitations ${ }^{1}$ and separating these two causes is the key to understanding the spin torque effect. To observe, identify, and understand where transformations stem from, high spatial resolution imaging of the domain wall spin structures is needed. One technique, which is particularly apt for such measurements is transmission electron microscopy, since very high spatial resolution $(<10 \mathrm{~nm})$ can be achieved. ${ }^{2}$ Elements have to be patterned on a very thin insulating (mostly $\mathrm{SiN}$ ) membrane with poor heat conductance. Therefore before this technique can be used to further study the spin torque effect, suitable cooling mechanisms have to be identified and implemented.

In this letter, we investigate current-induced domain wall motion due to the spin torque effect in permalloy nanowires defined on $\mathrm{SiN}$ membranes using transmission electron microscopy. By injecting current pulses domain wall motion and wall transformations are observed accompanied by significant heating even at low current densities. We identify the main cooling process as heat diffusion through the SiN substrate and exploit this by back coating the membrane with Al, which reduces local heating. Measurements on these samples reveal reduced heating and allow us to separate spin torque from heating effects by analyzing the different motions and transformations occurring.

Zigzag line structures (width: 100-500 nm, thickness: 10-24 nm, Permalloy) with three kinks were fabricated, ${ }^{10}$ as shown in Fig. 2. In one set of samples the $\mathrm{SiN}$ membrane substrates $(50 \mathrm{~nm}$ thick from Silson Ltd., UK) were additionally back coated with $5 \mathrm{~nm} \mathrm{Al}$.

We use off-axis electron holography to observe domain walls in those structures. ${ }^{11}$ A slightly simpler method is the Fresnel mode of Lorentz microscopy, ${ }^{12}$ which is used to identify the types and positions of the walls. The black and white contrast in those images originates from the deflected 


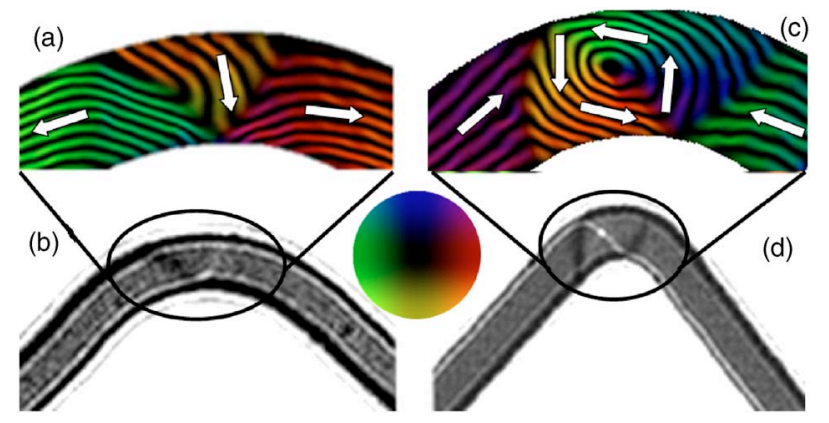

FIG. 1. (Color online) (a) Transverse domain wall imaged with off-axis electron holography (contour spacing $0.785 \mathrm{rad}$ ) and (b) in Lorentz mode (width: $560 \mathrm{~nm}$, thickness: $10 \mathrm{~nm}$ ). (c) Vortex domain wall imaged with electron holography (contour spacing of $1.57 \mathrm{rad}$ ) and (d) in Lorentz mode (width: $480 \mathrm{~nm}$, thickness: $20 \mathrm{~nm}$ ). The color code indicates the magnetization direction.

electron beam which is caused by the Lorentz force. ${ }^{13}$

Since Lorentz mode images do not directly reproduce the spin structure a first set of images was taken with both techniques to allow us to identify the wall type. The transverse domain walls result in a black and a white line, which correspond to the edges of the triangle with transverse magnetization [Figs. 1(a) and 1(b)]. The Lorentz images of vortex walls have two black lines parallel to each other in the direction transverse to the wire width and one white line connecting them from top to bottom with a brighter dot at the position of the vortex core [Figs. 1(c) and 1(d)]. The black and white contrast of vortex walls depends on the vortex circulation direction and inverts if the magnetization is inverted.

A pulse generator is used to inject current pulses with $10 \mu$ s duration. Increasing the current densities from 0 in steps of about $2 \times 10^{10} \mathrm{~A} / \mathrm{m}^{2}$ allows us to determine the critical current density for wall movement. During the pulse injection the resistance of the sample is monitored to determine the heating as a function of current density. ${ }^{14}$

We first consider a set of structures with no Al back layer (sample 1: width: $480 \mathrm{~nm}$, thickness: $24 \mathrm{~nm}$ ). We magnetize the zigzag permalloy lines with a field perpendicular to the wire by tilting the sample and applying a field of about $1 \mathrm{~T}$ with the objective lens. During this procedure domain walls are induced in the wire kinks. ${ }^{6}$ Therefore, before pulsing the electric current, we can observe three domain walls. After applying a pulse with a current density of 1.38 $\times 10^{11} \mathrm{~A} / \mathrm{m}^{2}$ two additional domain walls have been nucleated. Pictures of those regions where domain walls are found are overlayed on an optical microscope image of the whole permalloy wire in Fig. 2. This observed nucleation of domain walls is a thermally induced effect and has been reported previously. ${ }^{14}$ Further pulse injections arbitrarily nucleate and annihilate domain walls.

In a thinner structure (sample 2: width: $350 \mathrm{~nm}$, thickness: $10 \mathrm{~nm}$ ) we obtain transverse walls at the kinks after magnetization [Fig. 3(a)]. After a pulse injection of $j=3.14$ $\times 10^{11} \mathrm{~A} / \mathrm{m}^{2}$ we observe a transformation to a vortex wall spin structure [Fig. 3(b)]. This again can be understood in the context of heating because the domain wall undergoes a thermally activated transformation to its energetically favorable spin structure which is the vortex wall type for this geometry. ${ }^{1}$ Pulsing again with the same polarity results in random movement of the vortex walls, back and forth. This movement of the domain walls is not due to the spin torque

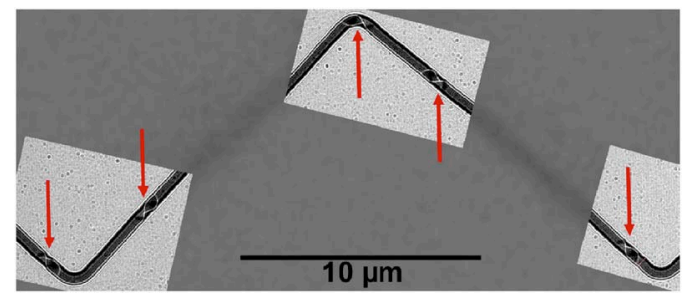

FIG. 2. (Color online) Lorentz images taken at those regions where domain walls are observable overlayed on an optical microscope image of the permalloy wire (sample 1: width: $480 \mathrm{~nm}$, thickness: $24 \mathrm{~nm}$ ). After magnetizing the wire with a field in the transverse direction, one domain wall is located at each corner. Applying a pulse with a current density of 1.38 $\times 10^{11} \mathrm{~A} / \mathrm{m}^{2}$ results in a nucleation of two more domain walls, so that five domain walls are now present as indicated by the arrows.

effect, but rather a thermally activated motion between different potential wells generated by the edge roughness and other defects. Togawa et al. ${ }^{15}$ measured the resistance rise in similar structures for similar pulse heights and they find heating close to, and even above, the Curie temperature (an increase in temperature of more than $350 \mathrm{~K}$ ) with a resistance increase of $100 \%$ for similar current densities as used here. Thus from our observations we can conclude that the observed effects can be attributed to heating.

We considered two possibilities to improve heat dissipation, given that heat can be transferred either into the substrate or along the wire and into the contacts. Calculations suggest that the former is more important, ${ }^{16}$ so to improve the heat flow through the substrate, we deposit $\mathrm{Al}$ on the back side of the membrane because of its high heat conductivity $(237 \mathrm{~W} /(\mathrm{K} \mathrm{m})$ compared to $18 \mathrm{~W} /(\mathrm{K} \mathrm{m})$ for pure $\mathrm{SiN}) .{ }^{17}$ Due to the large pulse length the conductance rather than the heat capacity is dominating the thermal properties since thermalization takes place within a few nanoseconds. We now inject in this sample current densities similar to what we used before (up to $j=4 \times 10^{11} \mathrm{~A} / \mathrm{m}^{2}$ ), but no changes occur. In such a sample (sample 3 with width of $580 \mathrm{~nm}$ and thickness of $12 \mathrm{~nm}$ ), first wall movements appear only at current densities of $7 \times 10^{11} \mathrm{~A} / \mathrm{m}^{2}$. These current densities are comparable to the critical current densities of similar permalloy structures on $\mathrm{Si}$ wafers, which provide a more efficient heat dissipation. ${ }^{6}$ This demonstrates an important point, namely, that observations of wall movements and transformations on SiN membranes at current densities far below what is reported on $\mathrm{Si}$ are due to thermal effects. Nominally identical polycrystalline permalloy wires should exhibit similar critical current densities for spin torque effects on all substrates. In this structure the domain walls move predominantly in the direction of the electron flow, as shown in Figs. 4(a)-4(d). Here four consecutive pulse injections move the walls similar distances with velocities be-
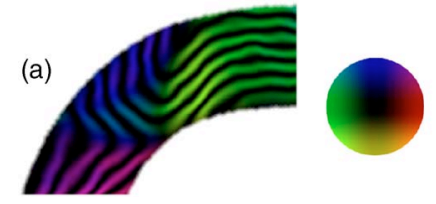

(b)

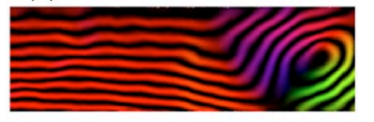

FIG. 3. (Color online) Magnetic induction maps (contour spacing of $0.785 \mathrm{rad})$ recorded using off-axis electron holography of domain walls in sample 2 (width: $350 \mathrm{~nm}$, thickness: $10 \mathrm{~nm}$ ). (a) After magnetization we obtain a transverse domain wall at the kink. (b) After a pulse injection with $j=3.14 \times 10^{11} \mathrm{~A} / \mathrm{m}^{2}$ a vortex wall spin structure in the straight part between two kinks is observed. 


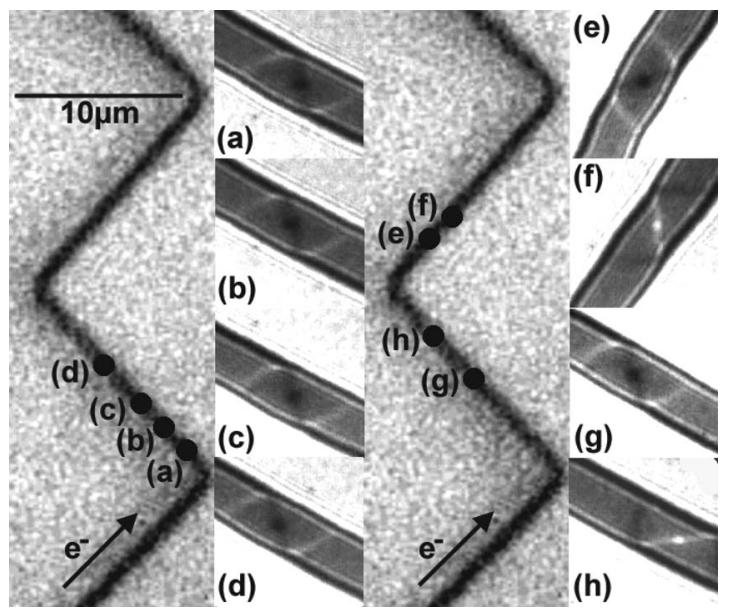

FIG. 4. Sample 3: Permalloy wire width: $580 \mathrm{~nm}$, thickness: $12 \mathrm{~nm}$. We apply pulses with a current density of $7 \times 10^{11} \mathrm{~A} / \mathrm{m}^{2}$. The Lorentz images are acquired in the alphabetical order with one $10 \mu$ s pulse between the adjacent pictures. [(a)-(d)] High resolution images of the same vortex wall that have moved in the direction of the electron flow from position [(a)-(d)] (as indicated above) during five consecutive pulses. Here, no change of the vortex circulation direction is observed. [(e)-(h)] Back and forth movement of the vortex domain wall with changes of the vortex circulation direction due to heating effects.

tween 0.05 and $0.3 \mathrm{~m} / \mathrm{s}$, in line with observations of similar wires on $\mathrm{Si}^{5,6} \mathrm{We}$ also note that the wall is always a vortex wall with a clockwise circulation direction. But this deterministic wall motion due to spin torque is still interjected by intervals with random thermal effects as visible in the sequence shown in Figs. 4(e) and 4(h). Here, the wall jumps randomly between different positions accompanied by a change in the vortex circulation direction. Such a change in the vortex circulation direction is not predicted by spin torque theory, where only transformations between transverse and vortex walls with one circulation direction occur. Statistical analysis of hundreds of pulse injections has shown that in samples with no Al, the walls move arbitrarily with and against the electron flow. No statistically significant motion in the electron flow direction was observed. In samples with $\mathrm{Al}$ coating we find for 299 pulse injections that statistically significantly more wall movements occur in the electron flow direction $(58.2 \%)$ than against it $(42.8 \%)$ and this was observed independently of the wall type (head-to-head or tail-to-tail wall), so that the only symmetry breaking of the motion direction is due to the spin torque effect. This means that here we observe a superposition of a randomizing heat effect and the spin torque, which induces a preference for wall motion in the electron flow direction. Furthermore we have not observed wall nucleation in this sample. Measurements of the resistance of these samples as a function of the current density yield a resistance increase of $20 \%$, which corresponds to an increase in temperature of $<150 \mathrm{~K}$ (as determined from measurements in a cryostat), meaning that the temperature stays below the Curie temperature in contrast to the samples without the $\mathrm{Al}$ back layer.

We can now conclude that the main channel of heat dissipation is via the substrate and this allows us also to understand why less heating was observed for thinner structures (arbitrary nucleation and annihilation of domain walls was only observed in sample 1 and neither in the thinner sample 2 nor in any $\mathrm{Al}$ coated samples). Thinner samples that have a larger interface with the substrate compared to the wire volume experience less heating and this trend was observed for all geometries. Therefore to prevent heating and observe spin torque effects, for a constant cross section, thinner wires are a better suited and even more cooling can be achieved by surrounding the wire with a good thermal conducting material.

We can identify a set of indicators to distinguish currentinduced domain wall motion effects due to spin torque from heating: (i) the domain wall motion is in the direction of the electron flow, (ii) the transformations occurring are compatible with those expected from theory and not only to energetically lower domain wall types, (iii) the critical current densities and the velocities for nominally identical structures should be similar even if the substrate is changed, and (iv) the temperature during current injection should stay far below $T_{C}$.

The authors acknowledge support by the Deutsche Forschungsgemeinschaft (SFB 513), Landesstiftung BadenWürttemberg, and the EPSRC(UK). One of the authors (F.J.) acknowledges support by the "Studienstiftung des Deutschen Volkes," another author (D.B.) acknowledges support by the European Union (Interreg III A program), and another one of the authors (R.E.D.B.) acknowledges support by the Royal Society.

${ }^{1}$ M. Laufenberg, D. Backes, W. Bührer, D. Bedau, M. Kläui, U. Rüdiger, C. A. F. Vaz, J. A. C. Bland, L. J. Heyderman, F. Nolting, S. Cherifi, A. Locatelli, R. Belkhou, and S. Heun, Appl. Phys. Lett. 88, 052507 (2006). ${ }^{2}$ M. Kläui, H. Ehrke, U. Rüdiger, T. Kasama, R. E. Dunin-Borkowski, D. Backes, L. J. Heyderman, C. A. F Vaz, J. A. C. Bland, G. Faini, E. Cambril, and W. Wernsdorfer, Appl. Phys. Lett. 87, 102509 (2005).

${ }^{3}$ M. Hayashi, L. Thomas, Ya. B. Bazaliy, C. Rettner, R. Moriya, X. Jiang, and S. S. P. Parkin, Phys. Rev. Lett. 97, 207205 (2006).

${ }^{4}$ L. Berger, J. Appl. Phys. 55, 1954 (1984).

${ }^{5}$ A. Yamaguchi, T. Ono, S. Nasu, K. Miyake, K. Mibu, and T. Shinjo, Phys. Rev. Lett. 92, 077205 (2004).

${ }^{6}$ M. Kläui, P.-O. Jubert, R. Allenspach, A. Bischof, J. A. C. Bland, G. Faini, U. Rüdiger, C. A. F Vaz, L. Vila, and C. Vouille, Phys. Rev. Lett. 95, 026601 (2005); Appl. Phys. Lett. 88, 232507 (2006); Phys. Rev. Lett. 94, 106601 (2005).

${ }^{7}$ G. Tatara and H. Kohno, Phys. Rev. Lett. 92, 086601 (2004).

${ }^{8}$ S. Zhang and Z. Li, Phys. Rev. Lett. 93, 127204 (2004).

${ }^{9}$ A. Thiaville, Y. Nakatani, J. Miltat, and Y. Suzuki, Europhys. Lett. 69, 990 (2005).

${ }^{10}$ D. Backes, L. J. Heyderman, C. David, R. Schäublin, M. Kläui, H. Ehrke, U. Rüdiger, C. A. F. Vaz, J. A. C. Bland, T. Kasama, and R. E. DuninBorkowski, Microelectron. Eng. 83, 17262006.

${ }^{11}$ R. E. Dunin-Borkowski, M. R. McCartney, and D. J. Smith, in Encyclopaedia of Nanoscience, Nanotechnology, edited by H. S. Nalwa (American Scientific, Stevenson Ranch, 2004), Vol. 3, pp. 41-100.

${ }^{12}$ J. N. Chapman, Mater. Sci. Eng., B 3, 355 (1989).

${ }^{13}$ J. Raabe, R. Pulwey, R. Sattler, T. Schweinböck, J. Zweck, and D. Weiss, J. Appl. Phys. 88, 4437 (2000).

${ }^{14}$ A. Yamaguchi, S. Nasu, H. Tanigawa, T. Ono, K. Miyake, K. Mibu, and T. Shinjo, Appl. Phys. Lett. 86, 012511 (2005).

${ }^{15}$ Y. Togawa, T. Kimura, K. Harada, T. Akashi, T. Matsuda, A. Tonomura, and Y. Otani, Jpn. J. Appl. Phys., Part 2 45, L683 (2006).

${ }^{16}$ C.-Y. You, I. M. Sung, and B.-K. Joe, Appl. Phys. Lett. 89, 222513 (2006).

${ }^{17}$ D. R. Linde, Handbook of Chemistry, Physics (CRC, Boca Raton, FL, 2001), Vol. 12, p. 219. 